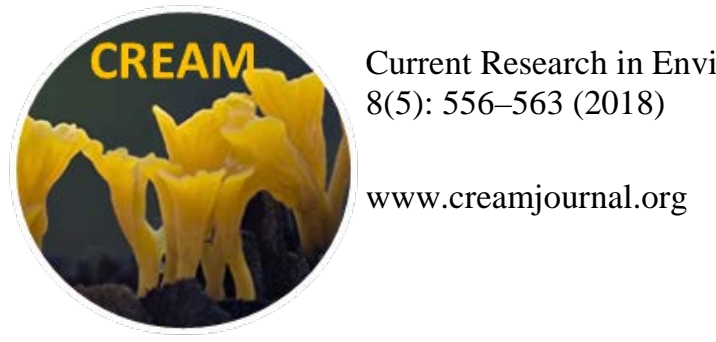

Current Research in Environmental \& Applied Mycology (Journal of Fungal Biology)

8(5): 556-563 (2018)

ISSN 2229-2225

\author{
Article \\ Doi 10.5943/cream/8/5/4
}

\title{
A preliminary study of wood-decay fungi in forests of northwest Arkansas
}

\author{
Alshammari $\mathbf{N}^{1,2}$, Stephenson $\mathrm{SL}^{1^{*}}$ \\ ${ }^{1}$ Department of Biological Sciences, University of Arkansas, Fayetteville, Arkansas USA \\ ${ }^{2}$ Department of Biological Sciences, University of Hail, Hail, Saudi Arabia
}

\begin{abstract}
Alshammari N, Stephenson SL 2018 - A preliminary study of wood-decay fungi in forests of northwest Arkansas. Current Research in Environmental \& Applied Mycology (Journal of Fungal Biology) 8(5), 556-563, Doi 10.5943/cream/8/5/4
\end{abstract}

\begin{abstract}
The present study represented an effort to characterize the assemblage of wood-decay fungi associated with forest ecosystems in northwest Arkansas. Specimens of fungi were collected from two different study areas. These were Pea Ridge National Military Park and Devil's Den State Park. In addition, small pieces of coarse woody debris (usually dead branches) were collected in the two study areas, returned to the laboratory and placed in plastic incubation chambers to which water was added. Fruiting bodies appearing in these chambers over a period of several months were collected and handled in the same manner as specimens on decaying wood obtained in the field. All specimens were identified through sequencing of the internal transcribed spacer (ITS) ribosomal DNA region. A total of 111 taxa were recorded, the majority of which could be identified to species. Seventy-seven taxa were recorded as field collections, whereas 34 taxa were recorded from the incubation chambers. Surprisingly, the two sets of data did not share any examples in common.
\end{abstract}

Key words - Basidiomycota - coarse woody debris - ITS ribosomal DNA region - Ozarks

\section{Introduction}

In forest ecosystems, certain fungi (usually referred to as wood-decay fungi) play an essential role in the decomposition of the coarse woody debris derived from the trees and other woody plants (e.g., shrubs) present. The decomposition of this coarse woody debris is a critical process, since it is important in nutrient recycling, soil development and the carbon budget of these ecosystems. For example, some wood-decay fungi have the capability to degrade the lignin component of coarse woody debris (Blanchette 1991, Eriksson et al. 2012), which otherwise would accumulate over time. Other wood-decay fungi meet their energy requirements by absorbing molecules derived the breakdown of the cellulose component of coarse woody debris. The fruiting bodies of wood-decay fungi also can serve as a source of food, nutrients and a breeding place for various animals (Stephenson 2010).

Wood-decay fungi are common in the forests of Arkansas, but there has never been an effort to characterize the assemblage of species present. The objective of the present study was to obtain data on wood-decay fungi in two different areas in northwest Arkansas. These two areas were Pea Ridge National Military Park and Devil's Den State Park. Pea Ridge National Military Park (36 $\left.27^{\prime} 15^{\prime \prime} \mathrm{N}, 94^{\circ} 02^{\prime} 05^{\prime \prime} \mathrm{W}\right)$, which has a total area of approximately 1740 ha, was established in 1956 to protect the site of the Civil War Battle of Pea Ridge, which was fought on 7-8 March 1862. 
The park is located in northwest Arkansas near the Missouri border. Forested areas of the park are dominated by a mixture of several species of oak (Quercus alba L., Q. velutina Lam., Q. stellata Wangenh. and Q. rubra L.) and hickory (Carya ovata [Mill.] K. Koch, C. texana Buckley and C. tomentosa Sarg.). Devil's Den State Park (3646’28” N, 94¹4’30” W), consists of about 1011 ha and is located near the border between Arkansas and Oklahoma. The park, which was constructed by the Civilian Conservation Corps during the 1930's, protects the largest sandstone crevice area in the United States. The forests present are similar in composition to those found in Pea Ridge National Military Park, but contains an admixture of species such as red maple (Acer rubrum L. and Acer saccharum Marshall) generally characteristic of relatively more mesic site conditions Stephenson et al. 2007).

\section{Materials \& Methods}

\section{Sampling for wood-decay fungi}

Each of the two study areas was visited on a number of occasions during the period of July to November 2017. Fruiting bodies of wood-decay fungi were located using an opportunistic search method as described by Cannon \& Sutton (2004). When fruiting bodies were observed, they were photographed in the field and then removed from the substrate upon which they occurred with the aid of a knife or a small hatchet. Fruiting bodies were then placed in a plastic collecting box with multiple compartments or loosely wrapped in aluminum foil and returned the laboratory. In the laboratory, specimens were dried at a temperature between $42-55^{\circ} \mathrm{C}$ on a food dehydrator, placed in plastic bags, and placed in the herbarium at the University of Arkansas (UARK) after being labeled with a unique collection number.

During a period extending from November 2017 to February 2018, the study areas were revisited and small pieces of coarse woody debris (usually dead branches) were collected. These were returned to the laboratory and placed in plastic incubation chambers $(33 \mathrm{~cm}$ long by $17 \mathrm{~cm}$ wide and $11 \mathrm{~cm}$ deep) along with a small amount of water. These were maintained for approximately two months, and water was added whenever necessary to keep the sample material moist. It was anticipated that in the incubation chambers, at least some of the mycelia already present in the coarse woody debris would produce fruiting bodies. When this happened, the fruiting bodies were collected and preserved in the same manner as those collected directly in the field.

\section{Samples and morphological observations}

Morphological aspects of the specimens collected in the field or from incubation chambers were determined with the use of an AmScope stereomicroscope (Gilbertson \& Ryvarden 1986, Sotome et al. 2013). Tentative identifications were based upon various morphological features such as the size, color and shape of the fruiting body and the presence or absence of such structures as a distinct cap or stipe. Identifications were made with the use of such sources of information as Gilbertson \& Ryvarden (1986, 1987), Bessette et al. (1997), Barron (1999), Binion et al. (2008), Elliott \& Stephenson (2018).

\section{DNA extraction, PCR and sequencing}

DNA was extracted from one or more representative specimens for each different taxon tentatively identified on the basis of morphological features of the fruiting body. This extraction was carried out using a Wizard ${ }^{\circledR}$ genomic purification kit (Promega Corporation, Madison, WI). Genomic DNA was amplified using the fungal-specific primers ITS1 (5'TCCGTAGGTGAACCTGCGG-3') and ITS4 (5'-TCCTCCGCTTATTGATATGC-3') (Toju et al. 2012). PCR amplifications were performed in a thermocycler programed for an initial denaturation at $95{ }^{\circ} \mathrm{C}$ for $5 \mathrm{~min}$, followed by 35 cycles of denaturation at $95^{\circ} \mathrm{C}$ for $45 \mathrm{sec}$, annealing at $50{ }^{\circ} \mathrm{C}$ for $45 \mathrm{sec}$, and extension at $72{ }^{\circ} \mathrm{C}$ for $1.5 \mathrm{~min}$, and a final extension at $72{ }^{\circ} \mathrm{C}$ for $10 \mathrm{~min}$. The length of amplified products was confirmed by $1 \%$ agarose gel electrophoresis gel using $0.5 \times$ TAE buffer, SYBR safe staining dye and $1 \mathrm{~kb}$ DNA ladder (New England Biolabs, Ipswich, MI). The amplicons 
were sent for Sanger sequencing to GeneWiz (South Plainfield, NJ). The sequences obtained from the latter company were cleaned up and then identified by doing nucleotide blast searches against the NCBI database (www.ncbi,nlm,nih,gov).

\section{Results}

For identification of specimens sequences were blasted against the NCBI database with a blast option to identify the taxon involved. At $97 \%$ sequence similarity, sequences were considered as identified to species when there was a match with an existing sequence; at a lower \% sequence identify, sequence were considered as identified only to genus. Although no single cutoff value has been universally established for species identification across the kingdom Fungi, 97\% sequence similarity has been used in a number of other studies (Raja et al. 2017). A total of 111 taxa (Table 1) were recorded, the majority of which could be identified to species. Seventy-seven taxa were recorded as field collections, whereas 34 taxa were recorded from the incubation chambers. Surprisingly, the two sets of data did not share any examples in common.

The taxa identified belong to at least 37 different families, with representatives of the Polyporaceae, Mycenaceae, Marasmiaceae, Pluteaceae, Steccherinaceae, Stereaceae and Xylariaceae the most common. Twenty taxa belonged to just the Polyporaceae. Mycena was the genus represented by the most species (7). Although the focus of this study was directed towards wood-decay fungi, some of the taxa identified have a different ecological role and were simply associated with decomposing wood. For example, such is the case for Cordyceps confragosa, which is an entomopathogenic fungus.

Table 1 Taxa of wood-decay fungi recorded from northwest Arkansas. Note: \%ID = percent sequence identity, $\mathrm{F}=$ field collection, $\mathrm{I}=$ incubation chamber collection and SGB $=$ sequence in GenBank.

\begin{tabular}{|l|l|l|l|l|l|}
\hline \multicolumn{1}{|c|}{ Taxon } & \multicolumn{1}{|c|}{ Family } & \%ID & F & I & \multicolumn{1}{|c|}{ ANO } \\
\hline Antrodia serialis (Fr.) Donk & Fomitopsidaceae & $\mathbf{9 9}$ & $\mathbf{X}$ & & KC585304.1 \\
\hline Auricularia americana Parmasto \& I. Parmasto & Auriculaceae & $\mathbf{9 9}$ & $\mathbf{X}$ & & JX065166.1 \\
\hline Bolbitius bisporus E.F. Malysheva & Bolbitiaceae & $\mathbf{9 9}$ & $\mathbf{X}$ & & NR153611.1 \\
\hline Byssomerulius incarnates (Schwein.) Gilb. & Meruliaceae & $\mathbf{9 9}$ & $\mathbf{X}$ & & MF773635.1 \\
\hline $\begin{array}{l}\text { Cladosporium cladosporioides (Fresen.) G.A. } \\
\text { de Vries }\end{array}$ & Davidiellaceae & $\mathbf{1 0 0}$ & $\mathbf{X}$ & & MF476049.1 \\
\hline Clitopilus hobsonii (Berk.) P.D. Orton & Entolomataceae & $\mathbf{9 9}$ & & X & FJ770395.1 \\
\hline $\begin{array}{l}\text { Coprinellus radians (Fr.) Vilgalys, Hopple \& } \\
\text { Jacq. }\end{array}$ & Psathyrellaceae & $\mathbf{1 0 0}$ & $\mathbf{X}$ & & KJ714004.1 \\
\hline Coprinellus sp. 1 & Psathyrellaceae & $\mathbf{9 9}$ & $\mathbf{X}$ & & KX611630.1 \\
\hline $\begin{array}{l}\text { Cordyceps confragosa (Mains) G.H. Sung, } \\
\text { J.M. Sung, Hywel-Jones \& Spatafora }\end{array}$ & Clavicipitaceae & $\mathbf{9 9}$ & & $\mathbf{X}$ & KT372853.1 \\
\hline Crucibulum laeve (Huds.) Kambly & Nidulariaceae & $\mathbf{9 8}$ & $\mathbf{X}$ & & DQ071701.2 \\
\hline $\begin{array}{l}\text { Cryptococcus yokohamensis Alshahni, Satoh \& } \\
\text { Makimura }\end{array}$ & Tremellaceae & $\mathbf{9 7}$ & & $\mathbf{X}$ & HM222928.1 \\
\hline $\begin{array}{l}\text { Daedaleopsis septentrionalis (P. Karst.) } \\
\text { Niemela }\end{array}$ & Polyporaceae & $\mathbf{9 9}$ & $\mathbf{X}$ & & HG973499.1 \\
\hline $\begin{array}{l}\text { Daedaleopsis tricolor (Bull.) Bondartsev \& } \\
\text { Singer }\end{array}$ & Polyporaceae & $\mathbf{1 0 0}$ & $\mathbf{X}$ & & KY235366.1 \\
\hline $\begin{array}{l}\text { Eurotium rubrum Jos. König, Spieck. \& W. } \\
\text { Bremer }\end{array}$ & Trichocomaceae & $\mathbf{1 0 0}$ & $\mathbf{X}$ & & EU001331.1 \\
\hline Exidia recisa (Ditmar) Fr. & Auriculariaceae & $\mathbf{9 9}$ & $\mathbf{X}$ & & LC098751.1 \\
\hline Exidia sp. 1 & Auriculariaceae & $\mathbf{9 9}$ & $\mathbf{X}$ & & MF161299.1 \\
\hline
\end{tabular}


Table 1 Continued.

\begin{tabular}{|c|c|c|c|c|c|}
\hline Taxon & Family & $\%$ ID & $\mathbf{F}$ & $\mathbf{I}$ & ANO \\
\hline $\begin{array}{l}\text { Fuscoporia gilva (Schwein.) T. Wagner \& M. } \\
\text { Fisch. }\end{array}$ & Hymenochaetaceae & 100 & $\mathbf{X}$ & & KU139196.1 \\
\hline Galiella rufa (Schwein.) Nannf. \& Korf & Sarcosomataceae & 99 & & $\mathbf{X}$ & AF485073.1 \\
\hline Ganoderma sp. 1 & Ganodermataceae & 99 & $\bar{X}$ & & AF255100.1 \\
\hline Gloeoporus dichrous (Fr.) Bres. & Meruliaceae & 100 & $\mathbf{X}$ & & JQ673109.1 \\
\hline Gymnopus dryophilus (Bull.) Murrill & Omphalotaceae & 99 & & $\mathbf{X}$ & DQ449974.1 \\
\hline Gymnopus earleae Murrill & Marasmiaceae & 99 & & $\mathbf{X}$ & DQ449994.1 \\
\hline Gymnopus foliiphilus R.H. Petersen & Omphalotaceae & 100 & $\mathbf{X}$ & & KY026721.1 \\
\hline Gymnopus luxurians (Peck) Murrill & Marasmiaceae & 99 & & $\mathbf{X}$ & AY256709.1 \\
\hline Gymnopus subnudus (Ellis ex Peck) Halling & Marasmiaceae & 99 & $\mathbf{X}$ & & KY026667.1 \\
\hline Hericium erinaceus (Bull.) Pers. & Hericiaceae & 99 & $\mathbf{X}$ & & AY534583.1 \\
\hline Hohenbuehelia angustata (Berk.) Singer & Pleurotaceae & 100 & $\mathbf{X}$ & & MG383816.1 \\
\hline $\begin{array}{l}\text { Hydnochaete tabacina (Berk. \& M.A. Curtis ex } \\
\text { Fr.) Ryvarden }\end{array}$ & Hymenochaetaceae & 97 & $\mathbf{X}$ & & JQ279562.1 \\
\hline Hymenochaete pinnatifida Burt & Hymenochaetaceae & 100 & $\mathbf{X}$ & & KU975472.1 \\
\hline Hyphodermella rosae (Bres.) Nakasone & Phanerochaetaceae & 100 & $\mathbf{X}$ & & KT962555 \\
\hline Hypoxylon crocopeplum Berk. \& M.A. Curtis & Xylariaceae & 99 & $\mathbf{X}$ & & KU683962.1 \\
\hline Infundibulicybe gibba (Pers.) Harmaja & Tricholomataceae & 99 & $\mathbf{X}$ & & MG663274.1 \\
\hline Irpex lacteus (Fr.) Fr. & Steccherinaceae & 99 & $\mathbf{X}$ & & KT272411.1 \\
\hline Lachnum virgineum (Batsch) P. Karst. & Hyaloscyphaceae & 99 & & $\mathbf{X}$ & AB481268.1 \\
\hline Lenzites betulinus (L.) Fr. & Polyporaceae & 100 & $\mathbf{X}$ & & KY313640.1 \\
\hline Lycoperdon pyriforme Schaeff. & Agaricaceae & 100 & $\mathbf{X}$ & & MF161171.1 \\
\hline Marasmiellus juniperinus Murrill & Marasmiaceae & 99 & & $\mathbf{X}$ & NR_119582.1 \\
\hline Marasmius pulcherripes Peck & Marasmiaceae & 98 & & $\mathbf{X}$ & FJ917615.1 \\
\hline Marasmius sp. 1 & Marasmiaceae & 95 & & $\mathbf{X}$ & FJ917619.1 \\
\hline Microstoma floccosum (Sacc.) Raitv. & Sarcoscyphaceae & 99 & $\mathbf{X}$ & & AF026309.1 \\
\hline Mycena aurantiomarginata (Fr.) Quél. & Mycenaceae & 99 & & $\mathbf{X}$ & JF908479.1 \\
\hline Mycena haematopus (Pers.) P. Kumm. & Mycenaceae & 100 & & $\mathbf{X}$ & MF686517.1 \\
\hline Mycena inclinata (Fr.) Quél. & Mycenaceae & 99 & $\mathbf{X}$ & & DQ490645.1 \\
\hline Mycena leaiana (Berk.) Sacc. & Mycenaceae & 99 & $\mathbf{X}$ & & JF908376.1 \\
\hline Mycena sp. 1 & Mycenaceae & 95 & $\mathbf{X}$ & & MG748570.1 \\
\hline Mycena sp. 2 & Mycenaceae & 96 & & $\mathbf{X}$ & FJ917615.1 \\
\hline Mycena sp. 3 & Mycenaceae & 90 & $\mathbf{X}$ & & DQ490645.1 \\
\hline $\begin{array}{l}\text { Mycorrhaphium adustum (Schwein.) Maas } \\
\text { Geest. }\end{array}$ & Steccherinaceae & 100 & $\mathbf{X}$ & & JN710573.1 \\
\hline Nectria mariannaeae Samuels \& Seifert & Nectriaceae & 99 & & $\mathbf{X}$ & GU586835.1 \\
\hline Neofavolus alveolaris (DC.) Sotome \& T. Hatt. & Polyporaceae & 99 & $\mathbf{X}$ & & KP283508.1 \\
\hline Neofavolus sp. 1 & Polyporaceae & 99 & $\mathbf{X}$ & & KP283508.1 \\
\hline Neofavolus sp. 2 & Polyporaceae & 99 & $\mathbf{X}$ & & KP283508.1 \\
\hline Neofavolus sp. 3 & Polyporaceae & 99 & $\mathbf{X}$ & & KP283508.1 \\
\hline Nigroporus vinosus (Berk.) Murrill & Steccherinaceae & 100 & $\mathbf{X}$ & & JX109857.1 \\
\hline Panellus stipticus (Bull.) P. Karst. & Mycenaceae & 99 & $\mathbf{X}$ & & AB863032.1 \\
\hline Panus conchatus (Bull.) Fr. & Panaceae & 100 & & $\mathbf{X}$ & MH016880.1 \\
\hline Panus lecomtei (Fr.) Corner & Polyporaceae & 99 & & $\mathbf{X}$ & KP135329.1 \\
\hline
\end{tabular}


Table 1 Continued.

\begin{tabular}{|c|c|c|c|c|c|}
\hline Taxon & Family & \%ID & $\mathbf{F}$ & $\mathbf{I}$ & ANO \\
\hline $\begin{array}{l}\text { Panus neostrigosus Drechsler-Santos \& } \\
\text { Wartchow }\end{array}$ & Polyporaceae & 99 & & $\mathbf{X}$ & KU761235.1 \\
\hline Panus rudis Fr. & Polyporaceae & 99 & $\mathbf{X}$ & & KU863048.1 \\
\hline Phaeomarasmius erinaceus (Peck) Singer & Inocybaceae & 99 & $\mathbf{X}$ & & MG773816.1 \\
\hline $\begin{array}{l}\text { Phanerochaete pseudosanguinea Floudas \& } \\
\text { Hibbett }\end{array}$ & Phanerochaetaceae & 100 & $\mathbf{X}$ & & KP135097.1 \\
\hline Phlebiopsis flavidoalba (Cooke) Hjortstam & Phanerochaetaceae & 97 & $\mathbf{X}$ & & KX065956.1 \\
\hline Pholiotina aeruginosa (Romagn.) M.M. Moser & Bolbitiaceae & 97 & $\mathbf{X}$ & & KF515918.1 \\
\hline Pleurotus floridanus Singer & Bolbitiaceae & 100 & & $\mathbf{X}$ & MG819742.1 \\
\hline Pleurotus ostreatus (Jacq. ex Fr.) P. Kumm. & Pleurotaceae & 99 & & $\mathbf{X}$ & MH395969.1 \\
\hline Pleurotus sapidus Sacc. & Pleurotaceae & 100 & $\mathbf{X}$ & & KY962449.1 \\
\hline Pluteus cervinus (Schaeff.) P. Kumm. & Pleurotaceae & 99 & $\mathbf{X}$ & & KF306014.1 \\
\hline $\begin{array}{l}\text { Pluteus chrysophlebius (Berk. \& M.A. Curtis) } \\
\text { Sacc. }\end{array}$ & Pleurotaceae & 99 & $\mathbf{X}$ & & HM562125.1 \\
\hline Pluteus longistriatus (Peck) Peck & Pluteaceae & 99 & $\mathbf{X}$ & & MH211936.1 \\
\hline Pluteus petasatus (Fr.) Gillet & Pluteaceae & 100 & $\mathbf{X}$ & & KJ009707.1 \\
\hline Pluteus romellii (Britzelm.) Sacc. & Pluteaceae & 99 & & $\mathbf{X}$ & KM983699.1 \\
\hline Pluteus sp. 1 & Pleurotaceae & 95 & $\mathbf{X}$ & & KY346856.1 \\
\hline Polyporus tuberaster (Jacq. ex Pers.) Fr. & Polyporaceae & 97 & & $\mathbf{X}$ & KJ668474.1 \\
\hline Polyporus sp. 1 & Polyporaceae & 100 & $\mathbf{X}$ & & KU324794.1 \\
\hline Psathyrella sp. 1 & Psathyrellaceae & 94 & $\mathbf{X}$ & & KC992949.1 \\
\hline $\begin{array}{l}\text { Pseudochaete tabacina (Sowerby) T. Wagner } \\
\text { \& M. Fisch. }\end{array}$ & Hymenochaetaceae & 98 & $\mathbf{X}$ & & KJ140591.1 \\
\hline Resupinatus alboniger (Pat.) Singer & Pleurotaceae & 99 & & $\mathbf{X}$ & KU355368.1 \\
\hline Resupinatus applicatus (Batsch) Gray & Tricholomataceae & 99 & & $\mathbf{X}$ & KU355368.1 \\
\hline $\begin{array}{l}\text { Rhizomarasmius pyrrhocephalus (Berk.) R.H. } \\
\text { Petersen }\end{array}$ & Psathyrellaceae & 99 & $\mathbf{X}$ & & DQ097369.1 \\
\hline Schizophyllum commune Fr. & Schizophyllaceae & 99 & & $\mathbf{X}$ & EU853847.1 \\
\hline $\begin{array}{l}\text { Schizopora ovispora (Corner) Hjortstam \& } \\
\text { Ryvarden }\end{array}$ & Schizoporaceae & 100 & & $\mathbf{X}$ & KX857803.1 \\
\hline Scutellinia crinite (Bull.) Lambotte & Pyronemataceae & 99 & & $\mathbf{X}$ & AY220797.1 \\
\hline Simocybe serrulata (Murrill) Singer & Inocybaceae & 99 & $\mathbf{X}$ & & MF153085.1 \\
\hline $\begin{array}{l}\text { Simplicillium lanosoniveum (J.F.H. Beyma) } \\
\text { Zare \& W. Gams }\end{array}$ & Cordycipitaceae & 100 & & $\mathbf{X}$ & AB758126.1 \\
\hline Spongipellis pachyodon (Pers.) Kotl. \& Pouzar & Hapalopilaceae & 100 & $\mathbf{X}$ & & KP135302.1 \\
\hline Steccherinum bourdotii Saliba \& A. David & Steccherinaceae & 99 & $\mathbf{X}$ & & KY948818.1 \\
\hline $\begin{array}{l}\text { Steccherinum laeticolor (Berk. \& M.A. Curtis) } \\
\text { Banker }\end{array}$ & Steccherinaceae & 99 & $\mathbf{X}$ & & KY948823.1 \\
\hline $\begin{array}{l}\text { Steccherinum murashkinskyi (Burt) Maas } \\
\text { Geest. }\end{array}$ & Steccherinaceae & 99 & $\mathbf{X}$ & & FJ798705.1 \\
\hline Stereum complicatum (Fr.) Fr. & Stereaceae & 99 & $\mathbf{X}$ & & KU559368.1 \\
\hline Stereum ostrea (Blume \& T. Nees) Fr. & Stereaceae & 100 & $\mathbf{X}$ & & KU559366.1 \\
\hline Stereum sanguinolentum (Alb. \& Schwein.) Fr. & Stereaceae & 99 & & $\mathbf{X}$ & AY089730.1 \\
\hline Stereum sp. 1 & Stereaceae & 100 & $\mathbf{X}$ & & KJ832051.1 \\
\hline
\end{tabular}


Table 1 Continued.

\begin{tabular}{|c|c|c|c|c|c|}
\hline Taxon & Family & \%ID & $\mathbf{F}$ & I & ANO \\
\hline Stereum sp. 2 & Stereaceae & 99 & $\mathbf{X}$ & & KR135365.1 \\
\hline Stereum sp. 3 & Stereaceae & 99 & $\mathbf{X}$ & & KJ831876.1 \\
\hline Tetrapyrgos nigripes (Fr.) E. Horak & Marasmiaceae & 99 & $\mathbf{X}$ & & DQ449942.1 \\
\hline Trametes conchifer (Schwein.) Pilát. & Polyporaceae & 100 & $\mathbf{X}$ & & JN164988.1 \\
\hline Trametes versicolor (L.) Lloyd & Polyporaceae & 100 & $\mathbf{X}$ & & MG554226.1 \\
\hline Trametes villosa (Sw.) Kreisel & Polyporaceae & 99 & $\mathbf{X}$ & & JN164970.1 \\
\hline Trametopsis cervina (Schwein.) Tomšovský & Polyporaceae & 100 & $\mathbf{X}$ & & MG663240.1 \\
\hline Trichaptum biforme (Fr.) Ryvarden & Polyporaceae (?) & 100 & $\mathbf{X}$ & & MF773616.1 \\
\hline Trichoderma viride Pers. & Hypocreaceae & 99 & & $\mathbf{X}$ & KM458804.1 \\
\hline Trichoderma sp. 1 & Hypocreaceae & 99 & & $\mathbf{X}$ & AB872440.1 \\
\hline Truncospora ohiensis Berk. & Polyporaceae & 98 & & $\mathbf{X}$ & KT695324.1 \\
\hline Tyromyces galactinus (Berk.) Bondartsev & Polyporaceae & 100 & $\mathbf{X}$ & & KY948829.1 \\
\hline Tyromyces kmetii (Bres.) Bondartsev \& Singer & Polyporaceae & 99 & $\mathbf{X}$ & & KF698747.1 \\
\hline Urnula craterium (Schwein.) Fr. & Sarcosomataceae & 99 & $\mathbf{X}$ & & EU834222.1 \\
\hline Xeromphalina kauffmanii A.H. Sm. & Mycenaceae & 99 & $\mathbf{X}$ & & MG663296.1 \\
\hline Xylaria cornu-damae (Schwein.) Berk. & Xylariaceae & 99 & & $\mathbf{X}$ & AF163031.1 \\
\hline Xylaria hypoxylon (L.) Grev. & Xylariaceae & 100 & $\mathbf{X}$ & & U47841 \\
\hline Xylaria sp. 1 & Xylariaceae & 99 & $\mathbf{X}$ & & KU683962.1 \\
\hline Xylaria sp. 2 & Xylariaceae & 92 & & $\mathbf{X}$ & JQ761642.1 \\
\hline
\end{tabular}

\section{Discussion}

Although studies of the wood-decay fungi have been carried out in various localities throughout the world (e.g., Leiniger et al. 1997, Alemu 2013, Lyngdoh \& Dkhar 2014, Bhutia 2016), the one described herein apparently represents the first such effort in the forests of northwest Arkansas. Indeed, we are not aware of any comparable studies anywhere in the Ozark physographic province, which encompasses norhern Arkansas and the southern half of Missouri while also extending westward into northern Oklahoma and southeast Kansas. Consequently, the prelimary results reported in this paper set the stage for future more comprehensive studies.

The total number of taxa reported (111) clearly indicates that the species richness of the assemblage of wood-decay fungi in the general study is quite high. Some of the taxa recorded, including such examples as Mycena haematopus, Panellus stipticus, Pleurotus ostreatus, Schizophyllum commune, and Stereum complicatum are common and widespread wood-decay fungi. Other taxa had sequences that did not match anything in the GenBank database. This would suggest that they are either rare (i.e., have been described but not yet sequenced) or are possibly new to science.

The most unexpected result of the present study is that the set of taxa recorded as field collections and the set of taxa ecorded from the incubation chambers did not share any examples in common. Field collections of fruiting bodies and the pieces of coarse woody debris placed in the incubation chambers were obtained at somewhat different times of the year, with the collecting periods overlapping only in November. Moreover, the pieces of coarse woody debris tended to be portions of branches, whereas the majority of fruiting bodies obtained as field collections typically occurred on logs. Although wood-decay fungi do differ with respect to some aspects of their biology (Worrall et al. 1997), it is difficult to see how this would account for such a profound difference, especially since the second author has observed such taxa as Mycena haematopus, recorded only in incubation chambers in the present study, commonly fruiting upon large logs. Clearly, the conditions that exist within an incubation chamber are not the same as those that exist in the field, and this might be a factor accounting for the observed differences. Nevertheless, it 
seems exceedingly unlikely that these differences would persist as additional data from the two study areas become available.

The second author is not aware of any published study of wood-decay fungi in which incubation chambers have been used in efforts to document the taxa present at a particular locality. In the present study, the number of incubation chambers yielding fruiting bodies of fungi was quite high (56\%), and since the chambers were monitored on a regular basis, the fruiting bodies could be collected when they were still in excellent condition. This certainly was a major advantage, both in terms of making identification easier and increasing the chances of being able to extract "good" DNA.

The fungal biota (all groups) of northwest Arkansas and adjacent areas of the Ozarks is not especially well-documented, since the appropriate surveys have never been carried out (Swartz 1933) However, based the limited body of data currently available (e.g., Discover Life [www.discoverlife.com] and GBIF [www.gbif.org]), there are no previous records from the region for a number of the taxa listed in Table 1. It is anticipated that additional more comprehensive studies will yield numerous additonal records.

\section{Acknowledgements}

We are grateful to personnel of the Pea Ridge National Military Park Service for granting us permission to carry out field work. The research described herein was funded in part by the Slime Mold Project at the University of Arkansas.

\section{References}

Alemu F. 2013 - Assessment of wild mushrooms and wood-decay fungi in Dilla University, Main Campus, Ethiopia. International Journal of Advanced Research 1, 458-467.

Barron G. 1999 - Mushrooms of Northeast North America. Lone Pine Publishing, Vancouver, British Columbia.

Bessette AE, Bessette AR, Fischer DW. 1997 - Mushrooms of Northeastern North America. Syracuse University Press, Syracuse, New York.

Bhutia LP. 2016 - A preliminary survey on diversity of wood decay fungi from East Sillim. Journalof Myopathological Research 54, 255-261.

Binion DE, Stephenson SL, Roody WC, Burdsall HH et al. 2008 - Macrofungi Associated with Oaks of Eastern North America. West Virginia University Press, Morgantown, West Virginia.

Blanchette RA. 1991 - Delignification by wood-decay fungi. Annual Review of Phytopathology 29, 331-403.

Cannon P, Sutton B. 2004 - Microfungi on wood and plant debris. In: Mueller G, Bills G, Foster M, (eds.) Biodiversity of Fungi: Inventory and Monitoring Methods. Burlington, Massachusetts, Elsevier Academic Press. Pp. 217-239.

Elliott TF, Stephenson SL. 2018 - Mushrooms of the Southeast. Timber Press, Portland, Oregon.

Eriksson KEL, Blanchette RA, Ander P. 2012 - Microbial and Enzymatic Degradation of Wood and Wood Components. Springer-Verlag, Berlin/Heidelberg, Germany.

Gilbertson RL, Ryvarden L. 1986 - North American Polypores, Volume I. Fungiflora, Oslo, Norway.

Gilbertson RL, Ryvarden L. 1987 - North American Polypores, Volume II. Fungiflora, Oslo, Norway.

Leiniger TD AD, Wilson AD, Lester DG. 1997 - Hurricane Andrew damage in relation to wood decay fungi and insects in bottomland hardwoods of the Atchafalaya Basin, Louisiana. Journal of Coastal Research 13, 1290-1293.

Lyngdoh A, Dkhar MS. 2014 - Wood-rotting fungi in East Khasi Hills of Meghalaya, Northeast India, with special reference to Heterobasidion perplexa (a rare species-new to India). 
Current Research in Environmental \& Applied Mycology 4, 117-124, Doi 10.5943/cream/4/1/10

Raja HA, Miller AN, Pearce CJ, Oberlies NH. 2017 - Fungal identification using molecular tools: a primer for the natural products research community. Journal of Natural Products 80, 756-770.

Sotome K, Akagi Y, Lee SS, Ishikawa NK, Hattori T. 2013 - Taxonomic study of Favolus and Neofavolus gen. nov. segregated from Polyporus (Basidiomycota, Polyporales). Fungal Diversity 58, 245-266.

Stephenson SL. 2010 - The Kingdom Fungi: The Biology of Mushrooms, Molds, and Lichens. Timber Press, Portland, Oregon.

Stephenson SL, Adams HS, Huebner CD. 2007 - Upland forest vegetation of the Ozark Mountains in northwestern Arkansas. Rhodora 109, 197-221.

Swartz D. 1933 - Studies of Arkansas fungi. American Midland Naturalist 14, 714-719.

Toju H, Tanabe AS, Yamamoto S, Sato H. 2012 - High-coverage ITS primers for the DNA-based identification of ascomycetes and basidiomycetes in environmental samples. PloS One 7(7), e40863.

Worrall JJ, Anagnost SE, Zabel RA. 1997 - Comparison of wood decay among diverse lignicolous fungi. Mycology 89:199-219. 